\title{
Power generation with laterally packaged piezoelectric fine wires
}

\author{
Rusen Yang1, Yong Qin', Liming Dai ${ }^{2}$ and Zhong Lin Wang" ${ }^{1 \star ~}$
}

\begin{abstract}
Converting mechanical energy into electricity could have applications in sensing, medical science, defence technology and personal electronics ${ }^{1}$, and the ability of nanowires to 'scavenge' energy from ambient and environmental sources $^{2-4}$ could prove useful for powering nanodevices ${ }^{5-8}$. Previously reported nanowire generators ${ }^{9-11}$ were based on vertically aligned piezoelectric nanowires that were attached to a substrate at one end and free to move at the other. However, there were problems with the output stability, mechanical robustness, lifetime and environmental adaptability of such devices. Here we report a flexible power generator that is based on cyclic stretching-releasing of a piezoelectric fine wire that is firmly attached to metal electrodes at both ends, is packaged on a flexible substrate, and does not involve sliding contacts. Repeatedly stretching and releasing a single wire with a strain of $0.05-0.1 \%$ creates an oscillating output voltage of up to $\sim 50 \mathrm{mV}$, and the energy conversion efficiency of the wire can be as high as $6.8 \%$.
\end{abstract}

In existing piezoelectric nanowire generators, a zigzag electrode $\sim 50-100 \mathrm{~nm}$ above an array of $\mathrm{ZnO}$ nanowires ${ }^{9-11}$ is forced to move by external forces or disturbances, thus bending the nanowires and inducing a voltage. However, the deliberate rubbing together of the electrode and the nanowire array results in wear, increased contact resistance/instability, and infiltration of vapour and liquid. In the design reported here, a piezoelectric fine wire (PFW) lies flat on a flexible substrate and is fixed to electrodes at both ends (Fig. 1a). When the substrate bends and stretches the wire, a tensile strain of $0.05-0.1 \%$ is induced in the wire (see Fig. 1b and Supplementary Information), leading to a drop in the piezoelectric potential along the wire, and forcing electrons to flow along an external circuit to charge the wire. And when the substrate is released, electrons flow back in the opposite direction. Periodically bending and releasing the PFW therefore generates an alternating current, and generators based on multiple PFWs can be integrated to raise the output voltage. The entire structure is packaged inside a thin layer of insulating wax or flexible polymer to maintain its physical stability (Fig. 1c).

Electrical measurements have shown that effective single-wire generators (SWG) all exhibit Schottky behaviour at one end (Fig. 1d). The short-circuit current $\left(I_{\text {sc }}\right)$ and open-circuit voltage $\left(V_{\mathrm{oc}}\right)$ were measured to characterize the performance of an SWG. To verify that the measured signal was generated by an SWG rather than the measurement system, we have developed two criteria: the 'switching-polarity' test ${ }^{10}$ (Fig. 1e) and a number of 'linear superposition' tests (see Supplementary Information). When the current meter was forward connected to an SWG, a positive voltage/current pulse was recorded during fast stretching of the substrate (Fig. 2a), and a corresponding negative pulse for fast release (where 'fast' means an angular bending rate of $\sim 260^{\circ} \mathrm{sec}^{-1}$ at a radius of $2 \mathrm{~cm}$ for the substrate). For a single PFW with a diameter of $\sim 4 \mu \mathrm{m}$ and a length of $\sim 200 \mu \mathrm{m}$, the output voltage was $20-50 \mathrm{mV}$ and the output current was $400-750 \mathrm{pA}$. Although the heights of the current peaks for the stretch and release appear different, possibly because of different straining rates, the areas under the peaks remain the same (see Supplementary Information, Table S1); the total charges transported in the two processes also remain the same to within $5 \%$ (with the total being $\sim 1 \times 10^{8}$ electrons). Similar experiments have been done when the PFW is under compressive strain.

When the current meter was reverse connected (Fig. 1e), the voltage and current pulses were also reversed (Fig. 2b). The nonsymmetric output of the SWG before and after switching the polarity is likely caused by a bias current in the measurement system, which is added to the current generated by the SWG in the forward configuration, and subtracted from the generated current in the reverse configuration. The true signal generated by the SWG is an average of the magnitudes observed under forward and reverse configurations. Further measurements showed that at least one Schottky contact and a piezoelectric material are needed for the SWG to produce an output (see Methods). The stability of the SWG has been examined by stretching-releasing an SWG at 22 cycles per minute for 45 and $120 \mathrm{~min}$ (Fig. 2c). The output signal was relatively stable, although a slight declining trend was seen, which was caused by the fatigue of the rubber band used for transmitting the mechanical bending to the substrate.

The electrical output of the SWG also depends on the rate at which the strain deformation is introduced, with the voltage/current output for a fast stretch or release being significantly higher than the voltage/current output for a slow stretch or release (where 'slow' means an angular bending rate of $\sim 7^{\circ} \mathrm{sec}^{-1}$ at a radius of $2 \mathrm{~cm}$ for the substrate; see Supplementary Information, Figs S5,S6). A slow stretch or release generates a lower output over a longer period of time signal (Fig. 2d) and the total charges transported in a fast stretch/slow release and a slow stretch/fast release remain the same to within $4 \%$, which means that the leakage current is very small (see Supplementary Information, Tables S2,S3).

The efficiency of the SWG has been estimated. The total mechanical deformation energy stored in the PFW after stretching is $W_{\mathrm{m}}=1 / 2 E A\left(L-L_{0}\right)^{2} / L_{0}$, where $E$ is the elastic modulus, $L_{0}$ is the original length, $L$ is the length after straining and $A$ is the cross-sectional area. The total electric energy generated is $W_{\mathrm{e}}=\int V I \mathrm{~d} t$. The energy conversion efficiency $\left(W_{\mathrm{e}} / W_{\mathrm{m}}\right)$ of the PFW itself, excluding the substrate film, is up to $6.8 \%$ (see Supplementary Information).

The output voltage of the generators was enhanced by connecting them in series after $I-V$ measurements (Fig. 3a) and power output measurement to ensure they were connected with the correct polarity and sequence. The $I-V$ characteristic of two SWGs connected in series still showed Schottky behaviour, and

${ }^{1}$ School of Materials Science and Engineering, Georgia Institute of Technology, Atlanta, Georgia 30332-0245, USA, ${ }^{2}$ Department of Chemical and Materials Engineering, School of Engineering, University of Dayton, Ohio 45469, USA; *e-mail: zlwang@gatech.edu 
a

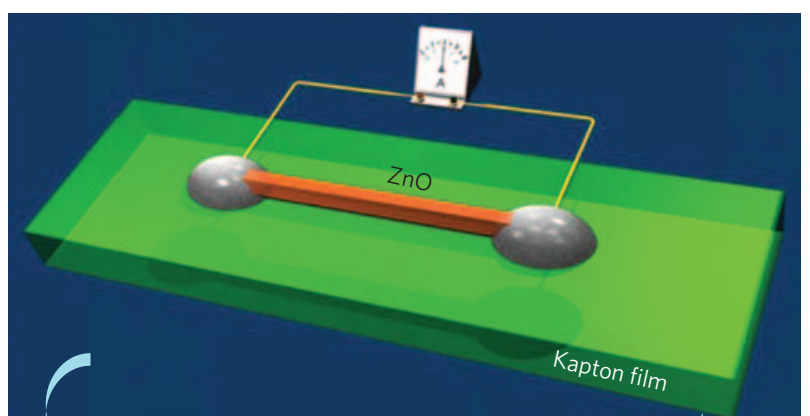

b

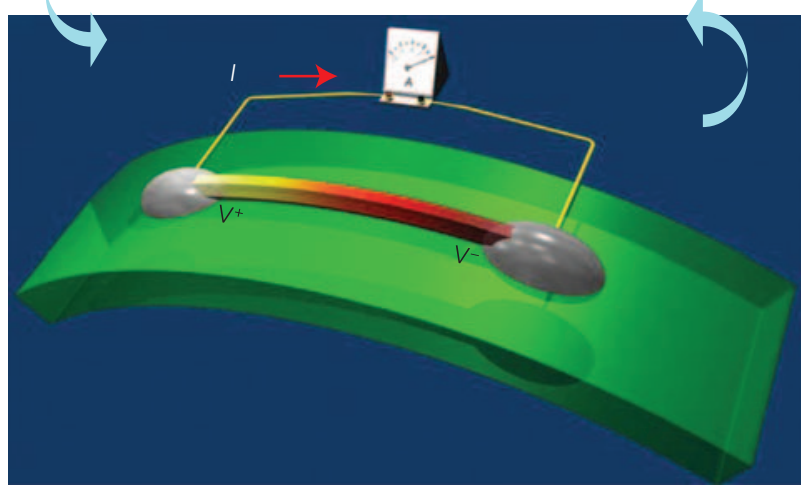

c

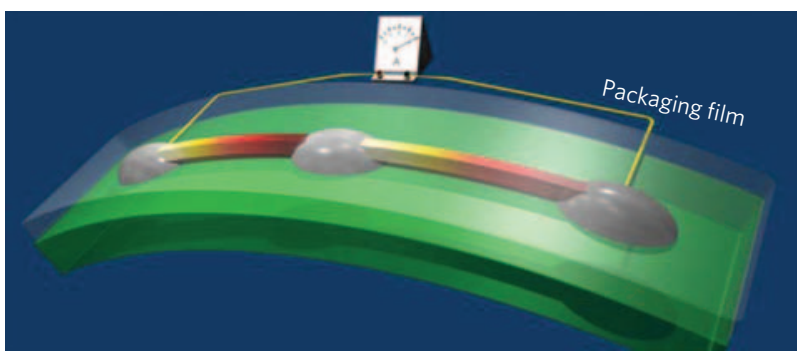

d

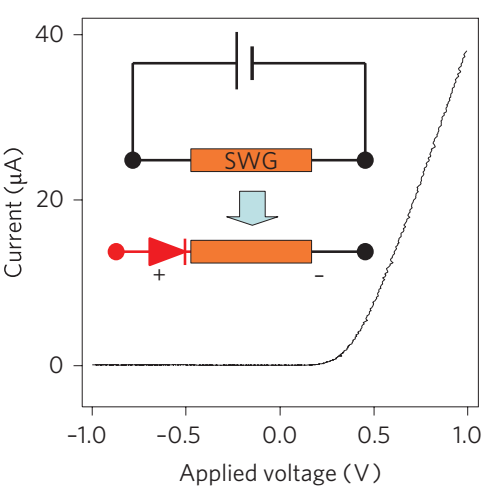

Figure 1 | Design of a piezoelectric fine wire (PFW) generator on a flexible substrate. a, The PFW lies on a polymer (Kapton) substrate, with both ends tightly bonded to the substrate and outlet interconnects. $\mathbf{b}$, Mechanical bending of the substrate creates tensile strain and a corresponding piezoelectric potential in the PFW, driving electrons through the external load. c, The wires are connected in series and packaged in a flexible polymer film. $\mathbf{d}, I-V$ characteristic of a single-wire generator (SWG), showing a typical Schottky diode characteristic with a forward-bias threshold voltage of $\sim 0.3 \mathrm{~V}$. We define the end-contact of the SWG that displays Schottky behaviour to be positive (shown here by a diode symbol at the interface). e, Definition of the switching-polarity test.

the output voltage was approximately the sum of the output voltages of the individual SWGs (Fig. 3b). When the two SWGs are connected in series but in reverse polarity, the output voltage is the difference of the two (Fig. 3c). We propose that satisfying the 'switching-polarity' and 'linear superposition' criteria is a necessary and sufficient condition to confirm that the output is indeed generated by the SWGs (see Supplementary Information). Finally, increasing the straining rate significantly increases the electrical output (Fig. 3d; see Supplementary Information, Figs S7,S8).

The $\mathrm{ZnO}$ PFWs used in our experiments were oriented along the $c$-axis. It is known that the (0001) and (0001) facets of $\mathrm{ZnO}$ are terminated with zinc and oxygen, respectively, and a non-symmetric Schottky contact usually appears at one end in transport measurement (see Supplementary Information). Figure 4 shows a proposed mechanism for the generation of current in single-wire generators in terms of the band structure of the system, with a Schottky barrier introduced to represent a high local contact resistance ( $\sim 50-1,000 \mathrm{M} \Omega$ ) to 'block' the flow of electrons through the wire (see Supplementary Information, Tables S4,S5).

When the PFW is subjected to tensile strain, a piezoelectric field is created in the PFW due to polarization of atoms in the crystal to create ionic charges (Fig. $4 \mathrm{~b}$; see Supplementary Information). These piezoelectric charges, which are non-mobile, remain in the PFW for an extended period of time without 'depletion' by the free carriers as long as the strain is preserved ${ }^{13,14}$. When the piezoelectric potential is positive $\left(V^{+}\right)$at the Schottky barrier side (which is on the left), and negative $\left(V^{-}\right)$at the ohmic side (which is on the right in Fig. 4a), the conduction band and Fermi level of the electrode on the right is raised by $\Delta E_{\mathrm{p}}=e\left(V^{+}-V^{-}\right)$with respect to the electrode on the left, and electrons will flow from the right electrode to the left electrode through an external load resistor because the resistance of the Schottky barrier is very high for voltages below a threshold value and thus blocks the flow of electrons through the wire (Fig. 4b). The electrons accumulate at the interfacial region between the left electrode and the wire, and this process continues until the potential created by the accumulated electrons balances the piezoelectric potential and the Fermi levels of the electrodes reach a new equilibrium value (Fig. 4c). When the tensile strain in the PFW is released, the immediate disappearance of the piezoelectric potential lowers the Fermi surface of the right electrode by $\Delta E_{\mathrm{p}}$, and the electrons that had accumulated near the left electrode flow back through the external circuit to the right electrode (Fig. 4d), returning the system to its original state. The peaks in the electrical output associated with the stretching and release are shown in Fig. 4e.

If the piezoelectric potential is negative $\left(V^{-}\right)$at the Schottky barrier side and positive $\left(V^{+}\right)$at the ohmic side (as a result of switching the $c$-axis orientation of the PFW), the Fermi level of the left electrode will be raised by $\Delta E_{\mathrm{p}}$ when the PFW is subjected to tensile strain, and electrons flow from left to right through the external load resistor (Fig. 4f). The free electrons can enter the PFW and screen the piezoelectric charges, reducing the local effective potentials to $V_{1}^{-}$and $V_{1}^{+}$, but the free electrons cannot completely neutralize/deplete the piezoelectric charges because the latter cannot move (Fig. 4g) ) $^{15,16}$. This process continues until an electric potential due to the free moving electrons is created across the PFW to balance the piezoelectric potential and the Fermi levels at the two electrodes reach a new equilibrium value (Fig. $4 \mathrm{~g}$ ). When the strain is released, the piezoelectric potential disappears, and the free charges used to screen the piezoelectric charges are free to move. At this moment, the Fermi level of the right electrode is higher than that of the left electrode, resulting in the electrons flowing back from right to left through the external circuit. This process ends when the Fermi levels of the two sides reach equilibrium again.

The Schottky barrier acts as a 'gate' that prevents the flow of the electrons through the PFW; in addition to preserving the piezoelectric potential, this also leads to the accumulation of electrons, which leads to a higher discharge rate. The Schottky barrier can either be at 

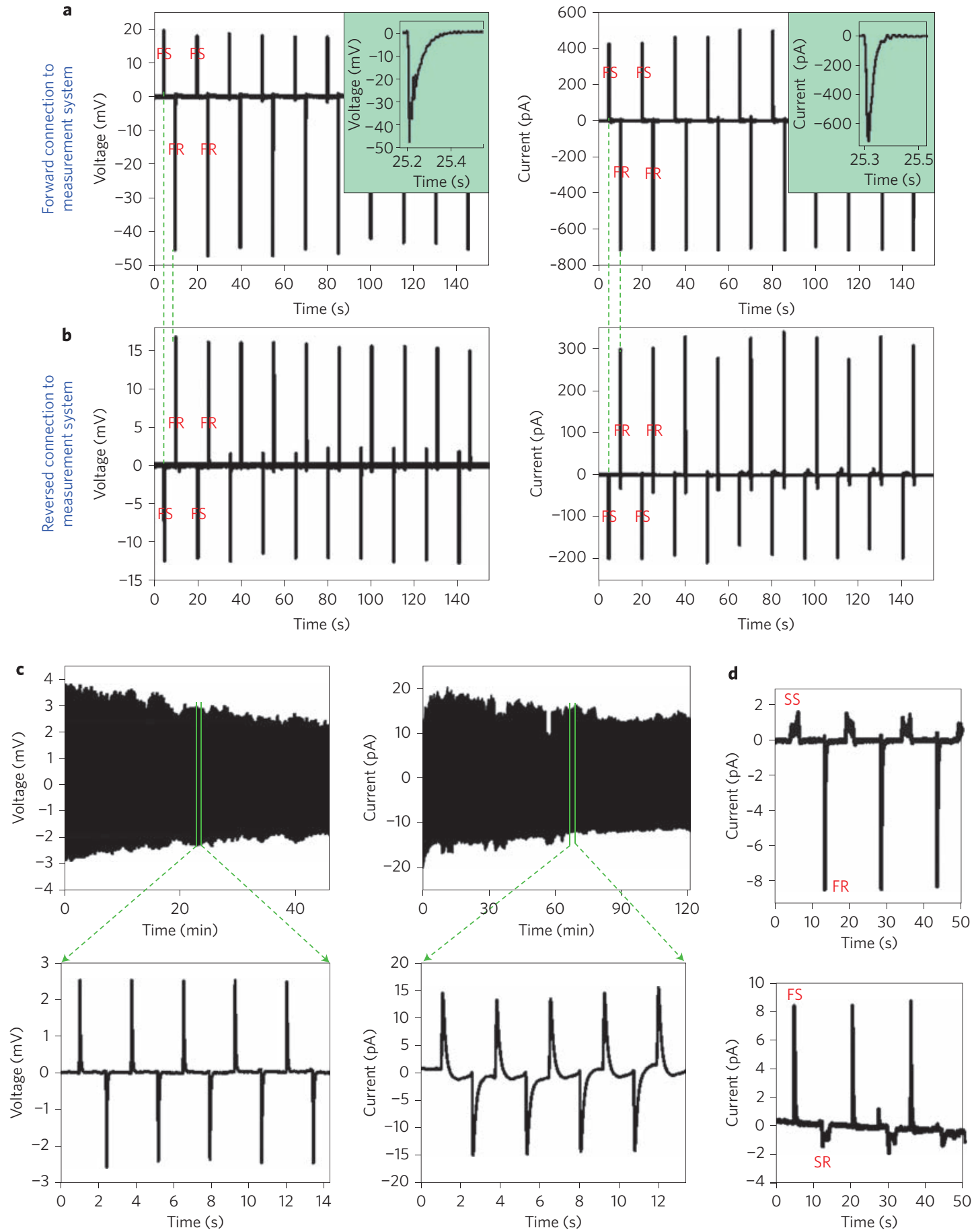

Figure 2 | Electrical output of a single-wire generator (SWG). a,b, Open-circuit voltage (left) and short-circuit current (right) of an SWG when subject to repeated cycles of fast stretching (FS) and fast release (FR) when forward-connected to the measurement system (a) and reverse-connected to the measurement system (b). The insets in a show the detailed shapes of peaks in the voltage and current outputs. c, The electrical output of a SWG operated at 22 cycles per minute demonstrates the stability of this approach. The bottom two panels show the detailed shapes of the voltage (left) and current (right) outputs. d, Electric current generated when the PFW was subject to slow stretching (SS) and fast release (top), and fast stretching and slow release (SR; lower curve). The data in $\mathbf{a}$ and $\mathbf{b}$ were acquired from the same SWG; the data in $\mathbf{c}$ and $\mathbf{d}$ are from different SWGs.

just one side of the PFW or at both sides. An insulator layer can also perform the same role (see Supplementary Information, Fig. S9). The PFW acts like a 'capacitor' and 'charge pump', storing electrons and then driving them back and forth through the external circuit as the PFW is stretched and released. This means that the generated voltage does not need to exceed a critical value (such as $0.4 \mathrm{~V}$ in
Fig. 3a) for the device to work. A repeated stretching-releasing of a single PFW creates a transient voltage up to $\sim 50 \mathrm{mV}$. The use of microwire/nanowires and their integration with a polymer film leads to a huge improvement in the mechanical flexibility and toughness of the entire generator (see Supplementary Information, Fig. S10). 

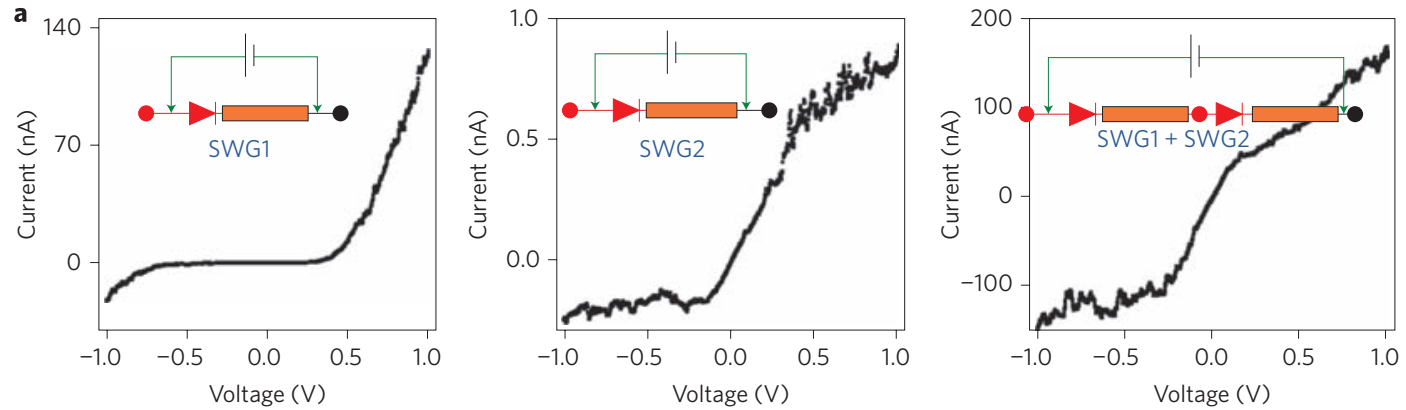

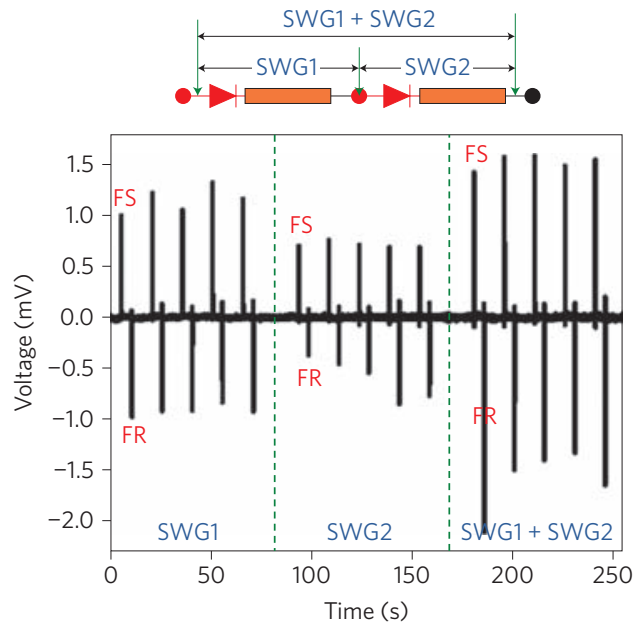

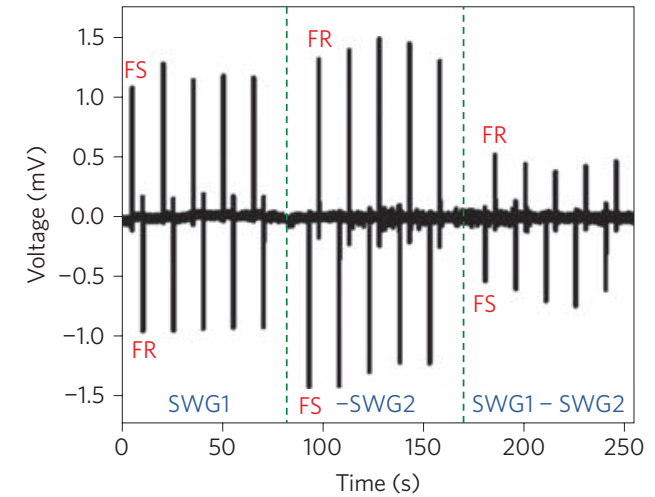
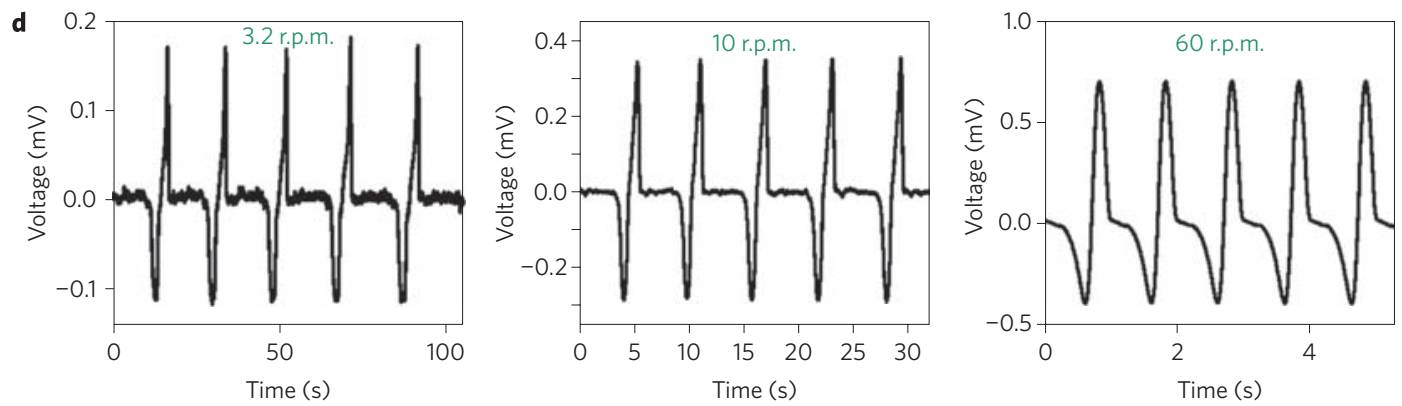

Figure 3 | Connecting two single-wire generators (SWGs) in series. a, $I-V$ characteristics of two individual SWGs (left, middle), and the same two SWGs in series (right). b,c, Voltage output of two individual SWGs subject to fast stretching and release, and (for times after $~ 180 \mathrm{~s}$ ) the same two SWGs connected constructively in series (b), showing that the output voltages are added, and then connected destructively in series (c), showing that the combined output is the difference of the two individual outputs. $\mathbf{d}$, As the number of stretch-release cycles per minute is increased from 3.2 (left) to 10 (middle) to 60 (right), the output voltage also increases, and is close to sinusoidal for 60 cycles per minute. The data in $\mathbf{a}, \mathbf{b}$ and $\mathbf{c}$ were acquired from the same two SWGs; the data in $\mathbf{d}$ are from a different SWG.

In summary, we have demonstrated a flexible power generator that has a number of advantages over generators based on vertically aligned nanowire arrays ${ }^{9,11}$ in terms of stability, robustness, cost, manufacturability and its ability to work in fluid and under harsh conditions. Moreover, the output voltage is 15-100 times higher than that of the d.c. nanogenerator ${ }^{9}$ and the fibre nanogenerator ${ }^{11}$, and there is scope to raise the power output by connecting individual generators in series or integrating thousands of such generators inside a common or multilayered substrate to form a flexible power sheet/film.

\section{Methods}

Fabrication of the generator. The generator was fabricated by bonding a $\mathrm{ZnO}$ PFW laterally on a Kapton polyimide film (Fig. 1a). The ZnO PFWs were synthesized by a physical vapour deposition method ${ }^{12}$, and typically have diameters of $3-5 \mu \mathrm{m}$ and lengths of 200 - $300 \mu \mathrm{m}$. We chose long PFWs because they were easy to manipulate, but the same process applies to nanowires. The thickness of the Kapton film received from DuPont Company was $50 \mu \mathrm{m}$. The film was first washed with acetone, isopropyl alcohol and ethanol under sonication. After that, the Kapton film was prepared for use as the substrate by dry cleaning using nitrogen gas and baking at $150{ }^{\circ} \mathrm{C}$ for $10 \mathrm{~min}$. The $\mathrm{ZnO}$ PFW was placed flat on the Kapton film using a probe station under an optical microscope. Silver paste was applied at both ends of the ZnO PFW to fix its two ends tightly on a flexible substrate (see Supplementary Information, Fig. S1). A current/voltage measurement meter was connected to two ends of the PFW without introducing any external power source in the circuit.

The role of the Schottky contact. To examine the role played by the Schottky contact for electricity generation, we purposely created symmetric ohmic contacts at two ends of a $\mathrm{ZnO}$ PFW using metallic indium. The output signals were very weak and did not correspondence to the frequency of the fast stretch and release of the substrate. More importantly, no reversal in output signal was observed in the switching-polarity test. To examine the role played by the $\mathrm{ZnO}$, we replaced the PFW 


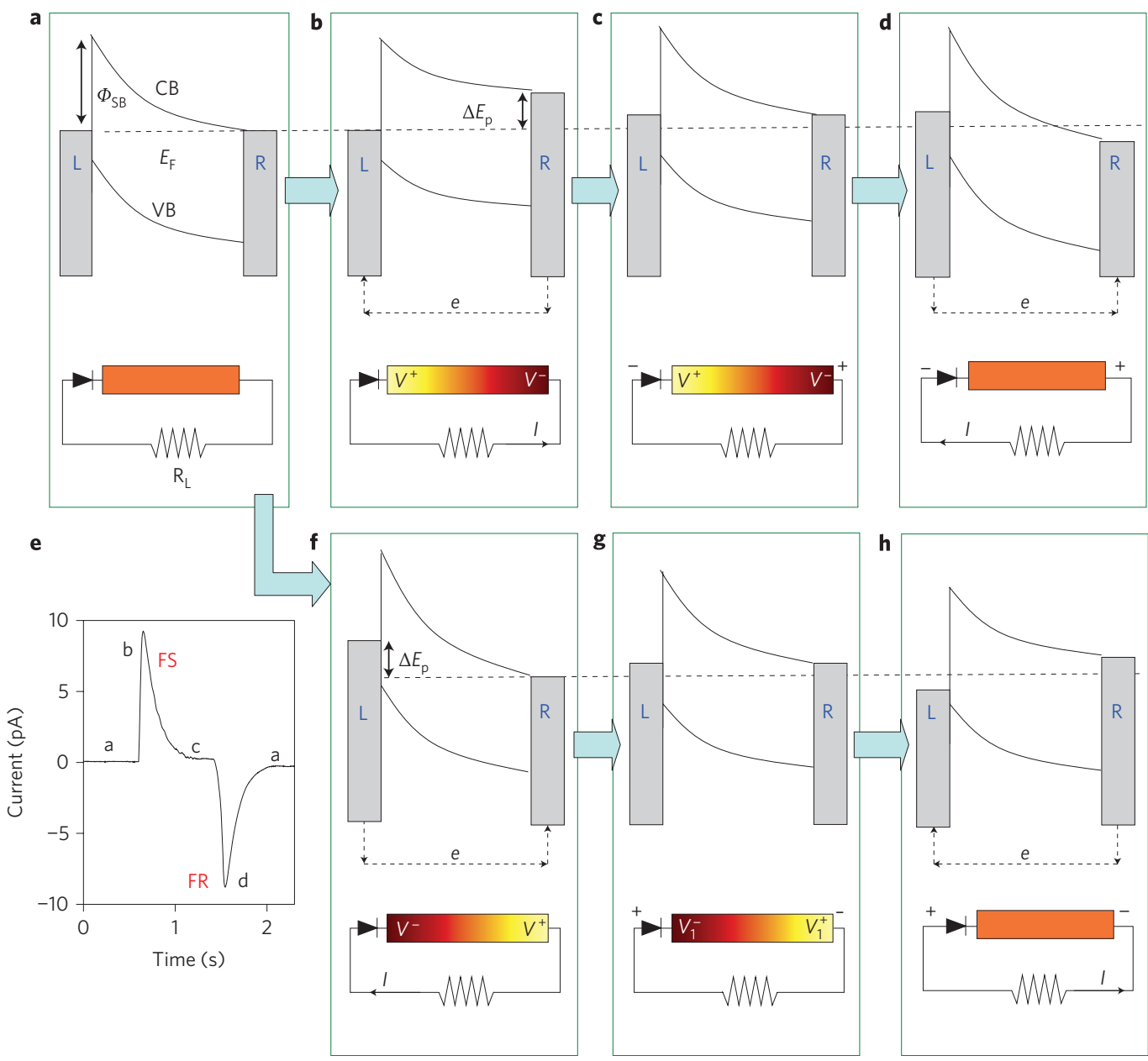

Figure 4 | Proposed mechanism for the generation of current in single-wire generators. a, Energy band diagram of a ZnO piezoelectric fine wire (PFW) making a Schottky contact with a metal electrode on the left and an ohmic contact with an electrode on the right: the dotted line is the Fermi level, $E_{\mathrm{F}}$, of the electrodes; $\mathrm{CB}$ and VB are the conduction and valence bands of the $\mathrm{ZnO}$; and $\Phi_{\mathrm{SB}}$ is the height of the Schottky barrier. A realistic measurement circuit containing a load resistor, $\mathrm{R}_{\mathrm{L}}$, that is much smaller than the resistance of the Schottky barrier and the PFW, is also shown. $\mathbf{b}$ - $\mathbf{d}$, The piezoelectric potential of the PFW is positive at the Schottky barrier side $V^{+}$, and negative at the Ohmic contact side $V^{-}$. When the PFW is tensile stretched, the Fermi levels of the two electrodes shift relative to each other as described in the text, generating a positive voltage/current peak as a result of electrons flowing from the right electrode to the left electrode (b), and they re-reach equilibrium (c), and they shift reversely again when the PFW is released (d), generating a negative voltage/current peak as the electrons flow back (from the left electrode to the right electrode). e, Experimentally measured output current from an SWG, showing the four different stages described above in $\mathbf{a}-\mathbf{d}$. $\mathbf{f}-\mathbf{h}$, When the piezoelectric potential of the PFW is negative at the Schottky barrier side $V^{-}$, and positive at the ohmic contact side $\mathrm{V}^{+}$, the Fermi levels shift in a different way during the stretch-release cycle, as described in the text.

by a carbon fibre, which is not piezoelectric, and found that no electricity was generated during the stretch-release cycle. We then coated a Kevlar fibre with a 500-nm-thick polycrystalline $\mathrm{ZnO}$ film. The contacts still maintained their ohmic behaviour, but no electricity was generated. This is because the $\mathrm{ZnO}$ nanocrystals in the film were randomly oriented without a measurable piezoelectric effect. After these studies (see Supplementary Information, Figs S2,S3,S4) we concluded that the presence of a Schottky contact at the interface and the piezoelectric property of the material are mandatory for SWGs. Further experiments showed that an SWG also produced output if both ends of the PFW were Schottky contacts.

\section{Received 23 June 2008; accepted 10 October 2008; published online 9 November 2008}

\section{References}

1. Paradiso, J. A. \& Starner, T. Energy scavenging for mobile and wireless electronics. IEEE Pervasive Comput. 4, 18-27 (January 2005).

2. Wang, Z. L. \& Song, J. H. Piezoelectric nanogenerators based on zinc oxide nanowire arrays. Science 312, $242-246$ (2006)

3. Hochbaum, A. I. et al. Enhanced thermoelectric performance of rough silicon nanowires. Nature 451, 163 - 167 (2008).
4. Tian, B. et al. Coaxial silicon nanowires as solar cells and nanoelectronic power sources. Nature 449, 885-890 (2007).

5. Patolsky, F. et al. Detection, stimulation and inhibition of neuronal signals with high-density nanowire transistor arrays. Science 313, $1100-1104$ (2006).

6. Javey, A., Guo, J., Wang, Q., Lundstrom, M. \& Dai, H. J. Ballistic carbon nanotube field-effect transistors. Nature 424, 654-657 (2003).

7. Bachtold, A., Hadley, P., Nakanishi, T. \& Dekker, C. Logic circuits with carbon nanotube transistors. Science 294, 1317 - 1320 (2001).

8. Chen, J. et al. Bright infrared emission from electrically induced excitons in carbon nanotubes. Science 310, $1171-1174$ (2005)

9. Wang, X. D., Song, J. H., Liu, J. \& Wang, Z. L. Direct-current nanogenerator driven by ultrasonic waves. Science 316, $102-105$ (2007)

10. Wang, X. D., Liu, J., Song, J. H. \& Wang, Z. L. Integrated nanogenerators in biofluid. Nano Lett. 7, 2475-2479 (2007).

11. Qin, Y., Wang, X. D. \& Wang, Z. L. Microfiber-nanowire hybrid structure for energy scavenging. Nature 451, $809-813$ (2008).

12. Pan, Z. W., Dai, Z. R. \& Wang, Z. L. Nanobelts of semiconducting oxides. Science 291, 1947 - 1949 (2001)

13. Liu, J. et al. Carrier density and Schottky barrier on the performance of DC nanogenerator. Nano Lett. 8, 328-332 (2008).

14. Zhou, J. et al. Mechanical-electrical triggers and sensors using piezoelectric microwires/nanowires. Nano Lett. 8, 2725-2730 (2008). 
15. Zhou, J. et al. Flexible piezotronic strain sensor. Nano Lett. 8, 3035-3040 (2008).

16. Zhou, J. et al. Piezoelectric-potential-controlled polarity-reversible Schottky diodes and switches of $\mathrm{ZnO}$ wires. Nano Lett. Doi: 10.1021/nl802497e (2008).

\section{Acknowledgements}

This research was supported by the US Department of Energy (Basic Energy Sciences), the National Science Foundation, the Emory-Georgia Tech Cancer Centre for Nanotechnology Excellence (funded by the National Institutes of Health) and the US Air Force Office of Scientific Research (FA9550-06-1-0384).

\section{Author contributions}

Z.L.W. and R.S.Y. designed the experiments. R.S.Y., Y.Q. and L.D. performed the experiments. Z.L.W. and R.S.Y. analysed the data and wrote the paper. All authors discussed the results and commented on the manuscript.

\section{Additional information}

Supplementary Information accompanies this paper at www.nature.com/

naturenanotechnology. Reprints and permission information is available online at http://npg. nature.com/reprintsandpermissions/. Correspondence and requests for materials should be addressed to Z.L.W. 